\title{
Robust conductive hydrogel with antibacterial activity and UV-shielding performance
}

Xiao Zhang ${ }^{a}$, Danting Sun ${ }^{a}$, Junqi Cai ${ }^{a}$, Weifeng Liu*a, Ning Yan ${ }^{c}$, Xueqing Qiu*b

${ }^{a}$ School of Chemistry and Chemical Engineering, Guangdong Engineering Research Center for Green Fine Chemicals, South China University of Technology, Wushan Road 381, Guangzhou, Guangdong, 510640, China

${ }^{b}$ School of Chemical Engineering and Light Industry, Guangdong University of Technology, Waihuan Xi Road 100, Guangzhou Higher Education Mega Center, Guangzhou, 510006, P.R China

c Department of Chemical Engineering and Applied Chemistry, University of Toronto, 200 College Street, Toronto, Ontario, M5S 3E5, Canada

*Corresponding authors

E-mail: weifengliu@scut.edu.cn (W. Liu); cexqqiu@scut.edu.cn_(X. Qiu).

This supporting information contains seven figures (Page 1-3) and two tables (Page 4).

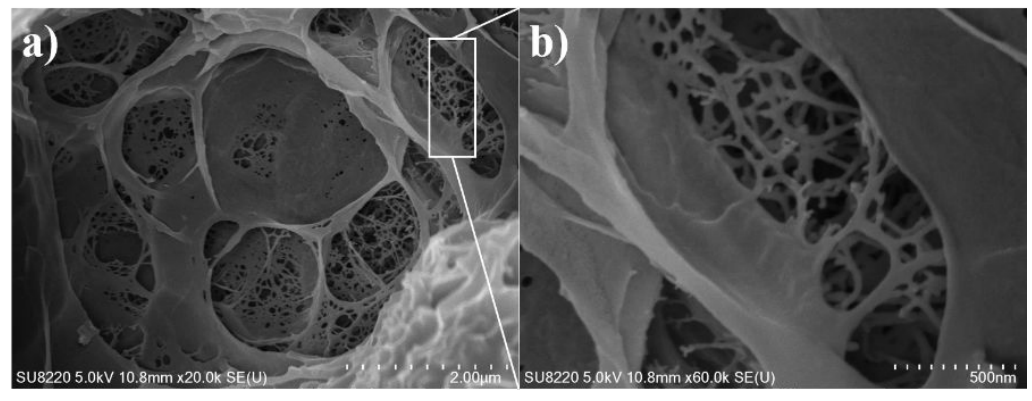

Figure S1. The SEM image of a) TA@LS-Ag/PVA composite hydrogel and b) its enlarged image. 


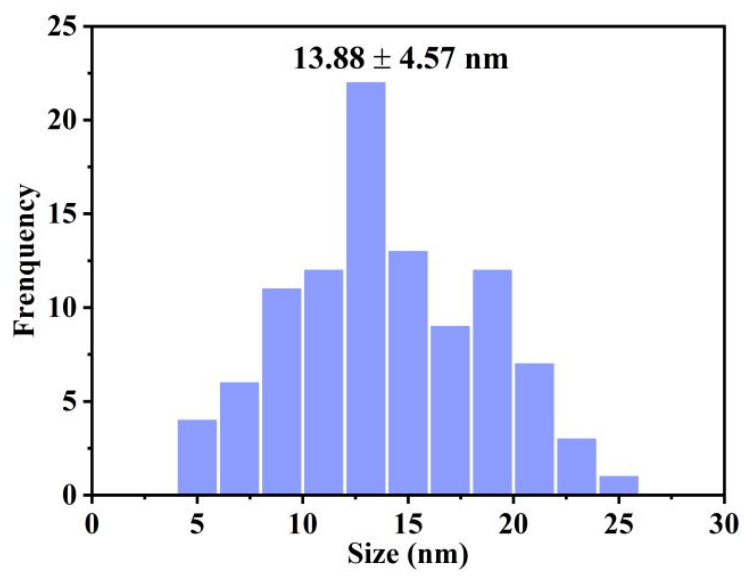

Figure S2. The particle size distribution of TA@LS-Ag in PVA composite hydrogel.
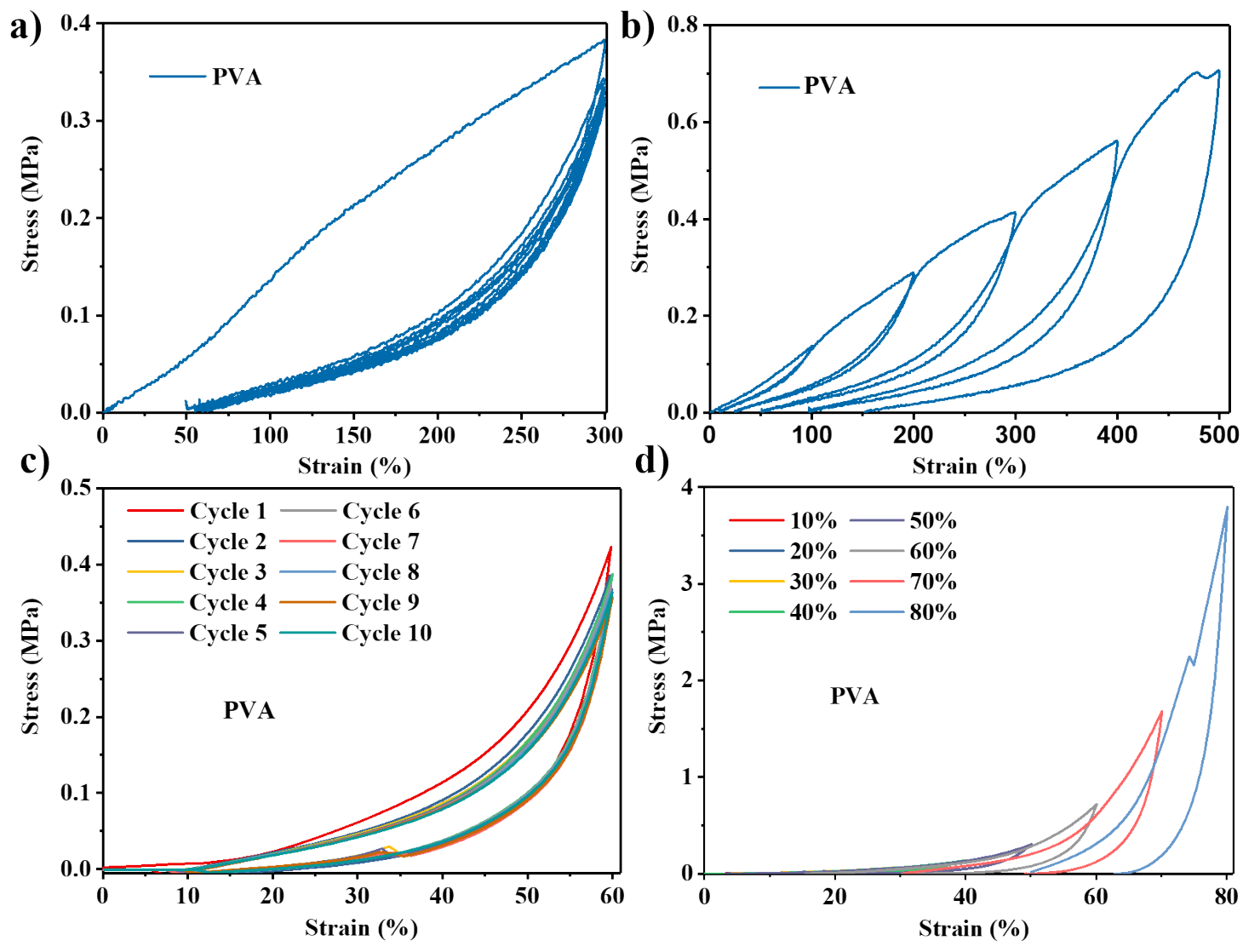

Figure S3. a) Continuous tensile loading-unloading curves at $300 \%$ strain of pure PVA hydrogel for 5 cycles without resting intervals. b) Successive tensile loading-unloading curves of pure PVA hydrogel under different strains $(100 \%, 200 \%, 300 \%, 400 \%$ and $500 \%)$. c) Continuous compressive loading-unloading curves at $60 \%$ strain of pure PVA hydrogel for 5 
cycles without resting intervals. d) Successive loading-unloading compression curves of pure PVA hydrogel under different strains $(10 \% \sim 80 \%)$.

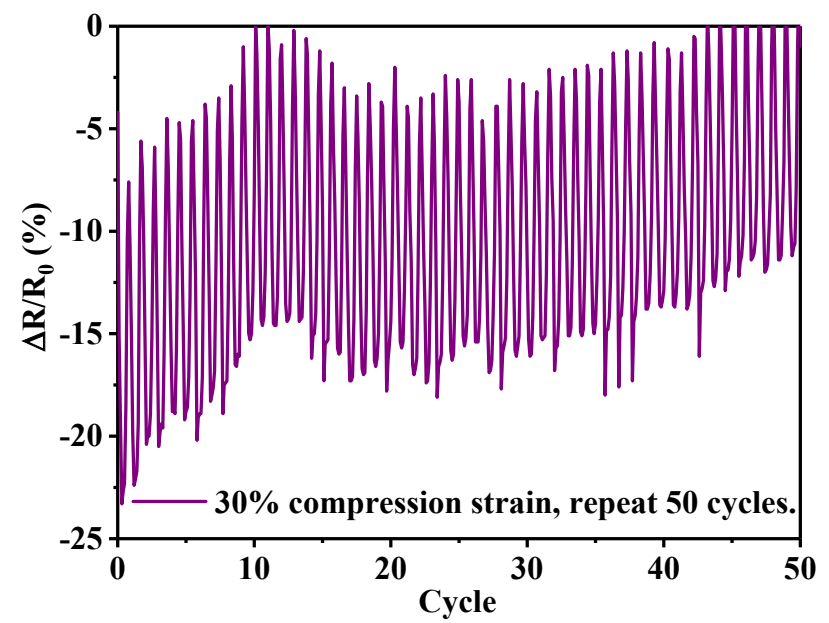

Figure S4. Resistance response of TA@LS-Ag/PVA composite hydrogel under repeated loading and unloading of $100 \%$ compressive strain for 100 cycles.

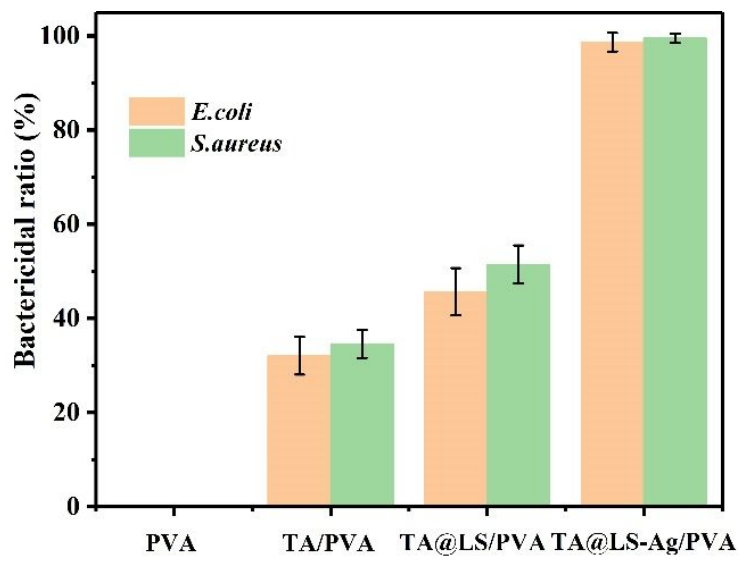

Figure S5. The bactericidal ratio of pure PVA hydrogel and PVA composite hydrogel. 
Table S1 MIC and MBC of varied samples.

\begin{tabular}{ccccc}
\hline \multirow{2}{*}{ Sample $^{\mathrm{a}}$} & \multicolumn{2}{c}{$\mathrm{MIC}^{\mathrm{b}} / \mathrm{ppm}$} & \multicolumn{2}{c}{$\mathrm{MBC} / \mathrm{ppm}$} \\
\cline { 2 - 5 } & E.coil & S.aureus & E.coil & S.aureus \\
\hline TA-Ag & $/$ & $/$ & $/$ & $/$ \\
LS-Ag & 30 & 30 & 60 & 60 \\
TA@LS & 15 & 15 & 30 & 30 \\
TA@LS-Ag $(4 / 1)$ & 7.5 & 7.5 & 15 & 15 \\
TA@LS-Agd $(2 / 1)$ & 3.8 & 3.8 & 7.5 & 7.5 \\
TA@LS-Agd $(1 / 2)$ & 60 & 60 & 120 & 120 \\
\hline
\end{tabular}

${ }^{a}$ The mass percentage of $\mathrm{AgNO}_{3}$ at all samples is $2 \mathrm{wt} \%$. ${ }^{\mathrm{b}} \mathrm{MIC}$ refers to minimum inhibitory concentration. ${ }^{\mathrm{c}} \mathrm{MBC}$ refers to minimum bactericidal concentration. ${ }^{\mathrm{d}}$ The radio refers to the mass ratio of TA to LS.

The ratio of TA to LS has an important influence on the antibacterial activity of the soaking agent TA@LS-Ag. In the case of TA only, the soaking agent TA-Ag performed no antibacterial activity because of the aggregation of TA. However, the antibacterial activity obviously improved after adding LS, demonstrating LS acted as a dispersant in TA@LS-Ag. Both the soaking agents LS-Ag and TA@LS exhibited a certain antibacterial activity resulted from the reducibility of LS and TA, which could reduce silver ions into silver nano particles in system. Excessive TA@LS-Ag (4/1) or deficiency amounts TA@LS-Ag (1/2) of TA reduced the antibacterial activity of the soaking agent, the best ratio is TA@LS-Ag (2/1). The data in Table S1 suggested the TA played the role of reducing agent in TA@LS-Ag, and LS played the role of reducing agent and dispersant.

Table S2. Mechanical properties of PVA and PVA composite hydrogels.

\begin{tabular}{cccccccc}
\hline Sample & $\begin{array}{c}\sigma_{\mathrm{t}}^{\mathrm{a}} \\
{[\mathrm{MPa}]}\end{array}$ & $\begin{array}{c}\varepsilon_{t}^{\mathrm{a}} \\
{[\%]}\end{array}$ & $\begin{array}{c}E_{t}^{\mathrm{a}} \\
{[\mathrm{MPa}]}\end{array}$ & $\begin{array}{c}\tau_{t}^{\mathrm{b}} \\
{\left[\mathrm{MJ} / \mathrm{m}^{3}\right]}\end{array}$ & $\begin{array}{c}\sigma_{c}{ }^{\mathrm{b}} \\
{[\mathrm{MPa}]}\end{array}$ & $\begin{array}{c}\tau_{c}{ }^{\mathrm{b}} \\
{\left[\mathrm{MJ} / \mathrm{m}^{3}\right]}\end{array}$ & $\begin{array}{c}\text { Conductivity } \\
{[\mathrm{S} / \mathrm{m}]}\end{array}$ \\
\hline PVA & $0.45 \pm 0.05$ & $334 \pm 13$ & $0.06 \pm 0.06$ & 0.72 & $4.39 \pm 0.06$ & 0.45 & 0.009 \\
LS/PVA & $1.38 \pm 0.08$ & $684 \pm 22$ & $0.21 \pm 0.05$ & 3.87 & $10.34 \pm 0.04$ & 0.82 & $/$ \\
TA/PVA & $2.89 \pm 0.06$ & $712 \pm 41$ & $0.29 \pm 0.03$ & 7.73 & $26.31 \pm 0.07$ & 2.37 & $/$ \\
TA@LS-Ag/PVA & $5.43 \pm 0.07$ & $1174 \pm 35$ & $0.24 \pm 0.05$ & 30.51 & $13.43 \pm 0.04$ & 1.24 & 4.1 \\
\hline
\end{tabular}

${ }^{a}$ Determined by tensile stress-strain curve, $\sigma_{t}, \varepsilon_{t}, E_{t}, \tau_{t}$ refer to the tensile stress, elongation at break, modulus and tensile toughness, respectively. ${ }^{\mathrm{b}}$ Determined by compression stress-strain curve, $\sigma_{\mathrm{c}}$, and $\tau_{c}$ refer to the compression stress at $90 \%$ compression strain and compression toughness, respectively. 